\title{
EVANS' SYNDROME IN AN OLD PATIENT: CASE PRESENTATION
}

\author{
Leonid I. DVORETSKY ${ }^{1 凶}$, Mariya A. KARNAUSHKINA ${ }^{1}$, Roza M. VAKOLYUK ${ }^{1}$, \\ Nelly V. DUBROVSKAYA ${ }^{1}$
}

${ }^{1}$ Department of Hospital Therapy No. 2, I.M. Sechenov First Moscow State Medical University, Moscow, Russian Federation

Received 16 Apr 2019, Corrections received 27 Aug 2019, Accepted 02 Oct 2019

https://doi.org/10.31688/ABMU.2019.54.4.27

\begin{abstract}
Introduction. Evans' syndrome (autoimmune hemolytic anemia combined with thrombocytopenia) is a rare hematological syndrome in the elderly.

Case report. A 94-year-old patient was diagnosed with Evans' syndrome with the secretion of monoclonal protein. The disease manifested initially by a periodic decrease in hemoglobin and platelet counts, accompanied by a deterioration of the clinical condition, and the development of a hemorrhagic syndrome. Long-term glucocorticoid therapy led to the development of osteoporosis, complicated by compression fracture of the lumbar vertebra. The main clinical manifestations of the disease (pain, limited mobility) were associated with the development of this complication. Conclusions. Diagnosis of Evans' syndrome in elderly patients requires the exclusion of other similar diseases. Long-term glucocorticoid treatment can cause complications, such as osteoporosis with a risk of vertebral fracture. The resulting complications can disrupt the quality of life to a much greater extent than the underlying disease. When choosing a treatment for elderly patients with Evans' syndrome, it is necessary to consider the possible benefits and the risk of complications.
\end{abstract}

\section{Résumé}

Syndrome d'Evans chez un patient âgé: rapport de cas

Introduction. Le syndrome d'Evans (anémie hémolytique auto-immune associée à une thrombopénie) est un syndrome hématologique rare chez les personnes âgées.

Rapport du cas. Un patient de 94 ans a été diagnostiqué avec le syndrome d'Evans avec la sécrétion de la protéine monoclonale. La maladie s'est accompagnée d'une diminution périodique des valeurs d'hémoglobine et de plaquettes, accompagnée d'une détérioration de l'état clinique, du développement d'un syndrome hémorragique. Un traitement prolongé aux glucocorticoïdes a conduit au développement de l'ostéoporose, compliquée par une fracture par compression de la vertèbre lombaire. Les principales manifestations cliniques de la maladie (syndrome douloureux, mobilité limitée) ont été associées au développement de cette complication.

Conclusions. Le diagnostic du syndrome d'Evans chez les patients âgés nécessite l'exclusion d'autres maladies s'apparentant au syndrome. Un traitement prolongé aux glucocorticoïdes peut entraîner des $\triangle$ Address for correspondence:
Leonid I. DVORETSKY

Department of Hospital Therapy No. 2, I.M. Sechenov First Moscow State Medical University, Moscow, Russian Federation

Address: Trubezkaja street no. 8, Moscow, Russia

Email:dvoretski@mail.ru 
Keywords: Evans' syndrome, autoimmune hemolytic anemia, immune thrombocytopenia, elderly.

\section{List of abbreviations:}

AHA - autoimmune hemolytic anemia, ITP - immune thrombocytopenia, ES - Evans' syndrome, GC - glucocorticoids, MP - monoclonal protein.

\section{INTRODUCTION}

In 1951, the American physician Robert Evans and his colleagues published the results of monitoring of 24 patients with isolated or combined autoimmune hemolytic anemia (AHA) and immune thrombocytopenia (ITP) ${ }^{1}$. Four of these patients were diagnosed with AHA in combination with ITP, without hemorrhagic manifestations; 6 patients with primary thrombocytopenic purpura, with no signs of hemolysis; 4 patients with AHA and thrombocytopenic purpura; in the remaining 10 cases, acquired isolated AHA or ITP were diagnosed. This observation and the same response of the monitored patients to splenectomy prompted the researchers to suggest the eventual presence of a common mechanism for the development of these hematological syndromes, in particular the role of formation of autoantibodies not only to red blood cells, which had already been known, but also to platelets. This hypothesis was confirmed by the presence of a factor agglutinating the platelets in the patients' serum, and some patients were found to have leukopenia, which could also be due to the presence of antibodies agglutinated to white blood cells. In later cases, AHA and ITP association were referred to as the Evans' syndrome (ES) and received a special code in the ICD-10, D69.3.J.

ES is a rare disease, occurring in $0.8-4.0 \%$ of patients with ITP and $\mathrm{AHA}^{2}$, although the exact incidence rate is unknown. In a study of 399 patients with AHA and 367 cases with ITP, ES has been diagnosed in only 6 patients out of $766^{3}$. According to another clinical and epidemiological study, ES was found in 7 patients among 164 children with ITP and 15 with $\mathrm{AHA}^{4}$. In a later study, ES was diagnosed in 23 patients out of 100 with AHA positive Coombs' test ${ }^{5}$. The most detailed analysis of this disease in adults is based on the observation of 68 patients with a mean age at the time of diagnosis of $55 \pm 3.3$ years $^{6}$. In $54.5 \%$ cases, AHA and ITP were diagnosed simultaneously, in $29.5 \%$ of patients ITP was preceded by complications telles que l'ostéoporose avec un risque de fracture vertébrale. Les complications qui en résultent peuvent perturber la qualité de vie dans une bien plus grande mesure que la maladie sous-jacente. Lors du choix d'un traitement pour les patients âgés atteints du syndrome d'Evans, il est nécessaire de prendre en compte les avantages possibles et le risque de complications.

Mots-clés: syndrome d'Evans, anémie immuno-hémolytique, thrombocytopénie immunitaire, personnes âgées.

AHA, while in $16 \%$, AHA was detected later ${ }^{6}$. In 10 patients (14.7\%), besides AHA and ITP, neutropenia was diagnosed ${ }^{6}$. The majority of patients $(96 \%)$ were found to have IgG antibodies to red blood cells (positive direct Coombs' test $)^{6}$. Almost half of the patients $(47 \%)$ required transfusion of red blood cells because of severe anaemia ${ }^{6}$. Hemorrhagic syndrome was observed in 44 patients $(64 \%)$, including 3 cases with life-threatening bleeding ${ }^{6}$.

Both AHA at ITP with ES feature the variability of cytopenia expression, progression (frequency of exacerbations and remissions) and response to treatment. Often, there are cases of severe hemolytic crisis, with eventual heavy bleeding caused by ITP. According to the previously mentioned study ${ }^{6}$, all 68 ES patients received GC, but 50 of them (73\%) required the use of a therapeutic alternative, i.e. splenectomy (19), and rituximab (11). With an average follow-up of 4.8 years, only 22 (32\%) patients were in remission without treatment. 16 patients (24\%) died. The causes of death were infection, malignant tumors, non-Hodgkin's lymphoma, cardiovascular disease, and myelodysplastic syndrome ${ }^{6}$.

\section{Case presentation}

A 94-year-old man was admitted to the hospital on March 10, 2016, reporting pain in the lumbar spine, aggravated by movement, bruising on limbs (mostly on the upper limbs), pain in ankle joints, swelling of feet, dizziness, unsteady gait, and dyspnea on little exertion. According to the history, the patient considers himself sick since 2005, when he first felt weakness, shortness of breath on exertion, and noticed bruises on skin. Peripheral blood study diagnosed anaemia and thrombocytopenia, which was not further surveyed and treated. Upon examination in 2015, along with anaemia and thrombocytopenia, the patient had a positive direct Coombs' test and was assigned glucocorticoid therapy (methylprednisolone $8 \mathrm{mg}$ ). The changes in hemoglobin, 


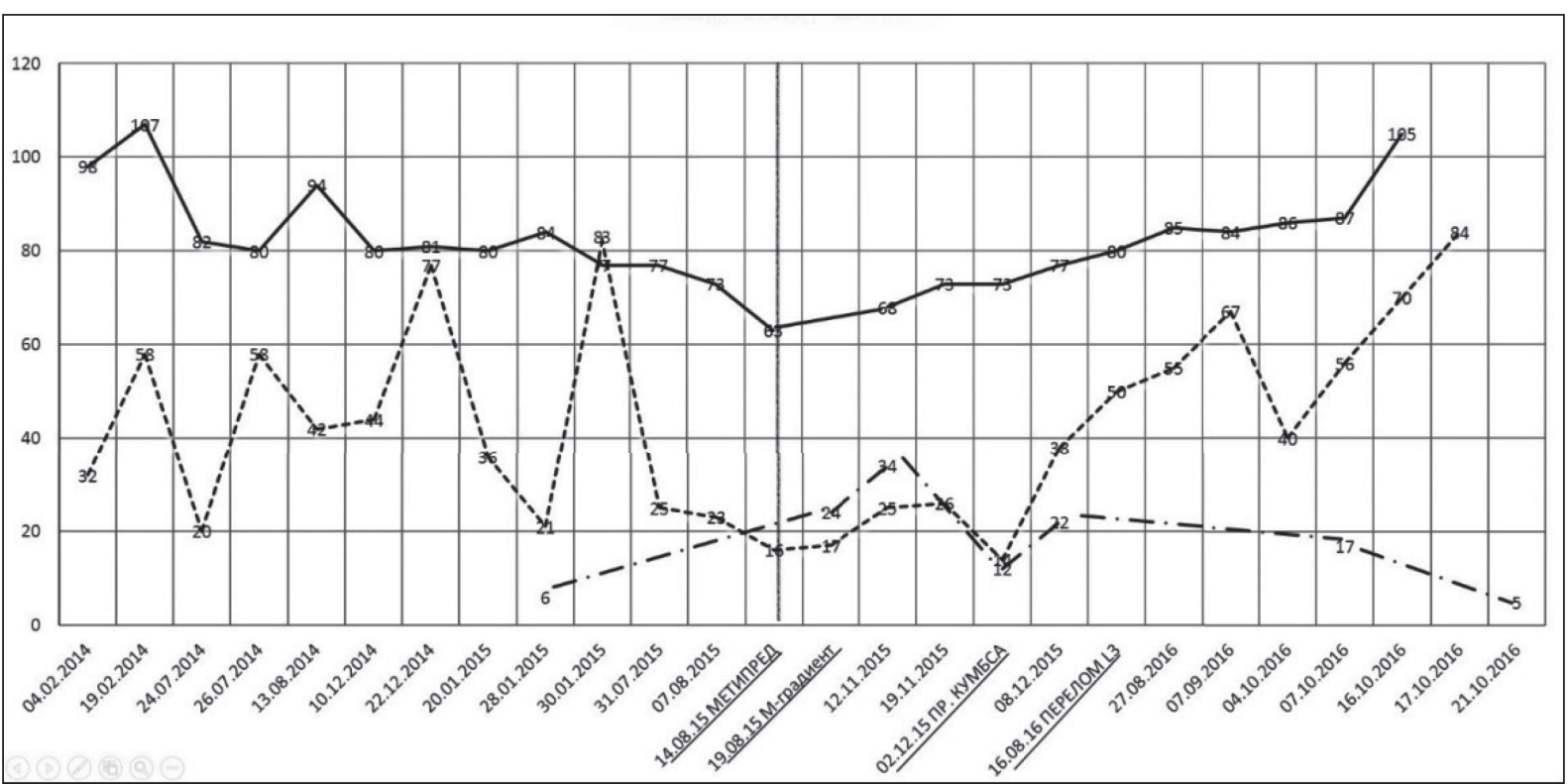

Fig. 1. Hemoglobin, platelet and reticulocyte counts in the patient over 2.5 years

Haemoglobin (g/l) (n x 10²/l); ------- Thrombocytes (n x 10\%/1); - - - Reticulocytes (\%o)

platelets, and reticulocytes values from 2014 to 2016 are shown in Fig. 1. In August 2016, he experienced a sharp pain in the lumbar spine (the patient denies falling), because of which the patient was not able to move independently. The X-ray diagnosed a compression fracture of L3. In October 2016, the patient was hospitalized with severe pain in the lumbar spine. The history shows that the patient has an implanted pacemaker and a prosthetic hip joint. No bad habits were reported. The patient did not have heredity or allergic history.

On admission, the condition was of moderate severity. The movements were limited because of pain, most of the time the patient stayed in bed. The skin was pale and jaundiced, with hemorrhagic lesions on forearms, thighs, and wrists (petechia, ecchymosis $0.5-1 \mathrm{~cm}$ in diameter). The peripheral lymph nodes were not enlarged. There was soreness under axial load on the spine. Cardiac sounds were normal, the blood pressure was $110 / 70 \mathrm{~mm} \mathrm{Hg}$, heart rate 60 $\mathrm{bpm}$. The breath rate was of $20 / \mathrm{min}$. Normal vesicular murmur, moist rales in the lower lungs. The abdomen was soft, painless to palpation in all areas, the liver was enlarged by $3 \mathrm{~cm}$, painless upon palpation, the spleen was enlarged by $2 \mathrm{~cm}$. The Pasternatsky's sign was negative on both sides.

Peripheral blood (October 04, 2016): Hb $8.6 \mathrm{~g} / \mathrm{d}$ L; RBC 3.37x10 $12 / \mathrm{L}$; MCH 25 pg/L; MCV 82; reticulocytes $37 \%$; Leukocytes $4.8 \times 10^{\%} / \mathrm{L}$; erytronormoblasts 2/100; platelets $40 \times 10^{9} / \mathrm{L}$; erythrocyte sedimentation rate (ESR) $8 \mathrm{~mm} / \mathrm{h}$.
Biochemical blood tests (October 04, 2016): total protein $55 \mathrm{~g} / \mathrm{L}$; albumin $30 \mathrm{~g} / \mathrm{L}$; BUN 13.10 $\mathrm{mmol} / \mathrm{L}$; creatinine $107 \mu \mathrm{mol} / \mathrm{L}$; total cholesterol 2 $\mathrm{mmol} / \mathrm{L}$; total bilirubin $55.9 \mu \mathrm{mol} / \mathrm{L}$; direct bilirubin $26.2 \mu \mathrm{mol} / \mathrm{L}$; AST $10 \mathrm{U} / \mathrm{L}$; ALT $18 \mathrm{U} / \mathrm{L}$; iron 13.7 $\mu \mathrm{mol} / \mathrm{L}$; lactate dehydrogenase $1200 \mathrm{U} / \mathrm{L}$; alkaline phosphatase $26.6 \mathrm{U} / \mathrm{L}$; gamma GT $11 \mathrm{U} / \mathrm{L}$; potassium $5.2 \mathrm{mmol} / \mathrm{L}$.

The patient's history contains results of additional studies: August 2015 - monoclonal protein (4.5\%) in a gamma-factions, typified as the monoclonal IgG-kappa; December 2015 - positive direct Coombs' test.

Ultrasonography of the abdomen: liver - the left lobe is not enlarged, the right lobe is enlarged to 160 $\mathrm{mm}$ (normal size $140 \mathrm{~mm}$ ), the contours are even, the structure is homogeneous; spleen - enlarged, $138 \times 57 \mathrm{~mm}$ (normal size $120 \times 60 \mathrm{~mm}$ ), the area is increased to $73 \mathrm{~cm}^{2}$ (the normal area $40 \mathrm{~cm}^{2}$ ), the structure is homogeneous. Spine CT scan: straightening of lumbar lordosis, moderate intervertebral osteochondrosis, with a decrease in height of L5-S1 disc, pronounced involuting osteoporosis L3 with a compression body fracture (Fig. 2).

Transthoracic echocardiogram showed decreased left ventricular systolic function with ejection fraction $46 \%$, thickened left ventricular wall, severely compromised right ventricle, right and left atria, high mitral regurgitation.

Coronary angiography was performed and showed chronic occlusion of middle-tract of left anterior descending artery with homocoronary collateral 

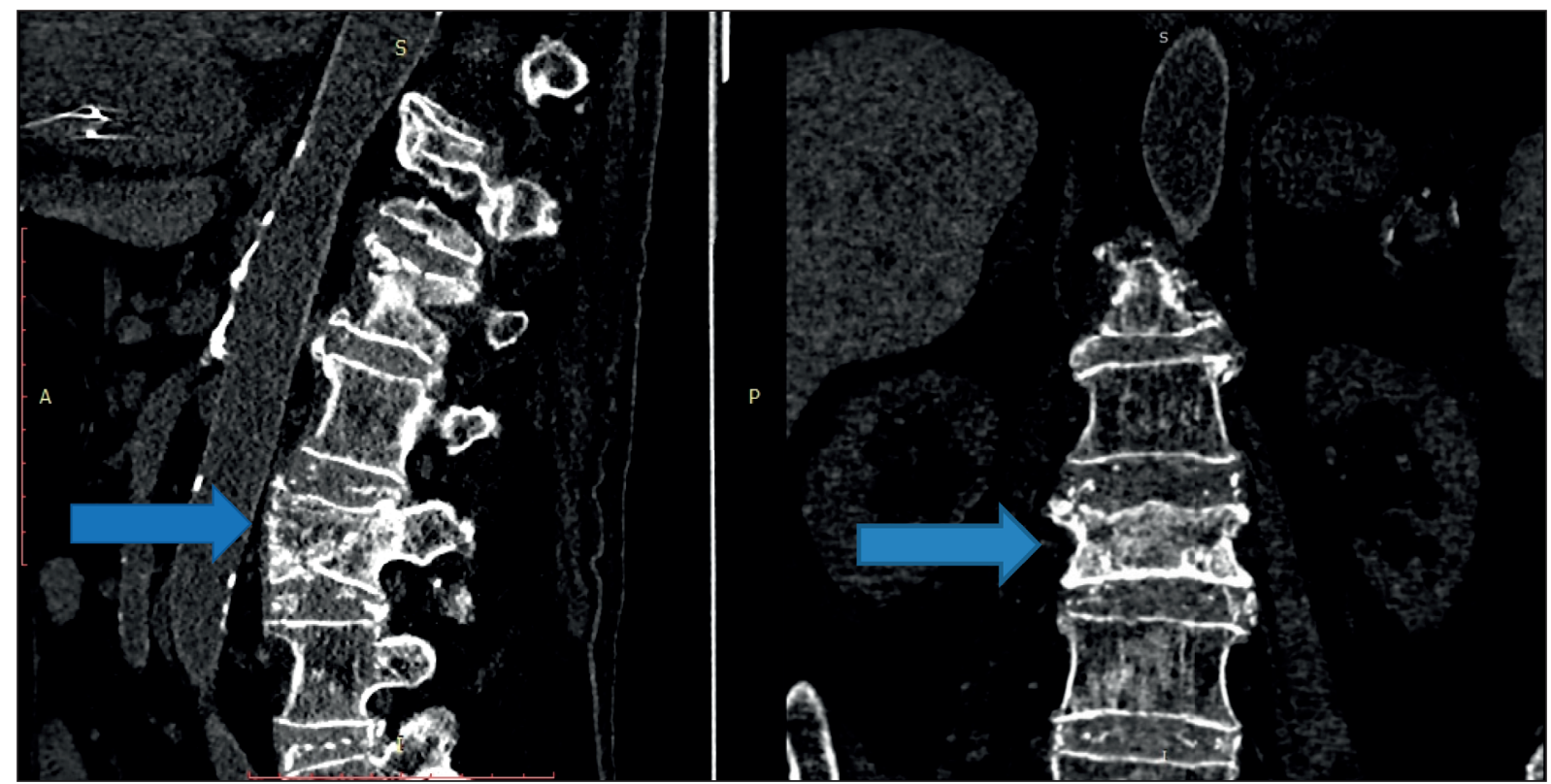

Fig. 2. Vertebral compression fracture in a patient who received glucocorticoid treatment

circulation from left circumflex artery, ectasic lesions of right coronary artery with sub occlusive stenosis $(75 \%)$ in the proximal tract of posterior descending artery.

Based on the history, clinical and laboratory examination findings, the patient had the following positive diagnosis: Coronary Heart Disease. Atherosclerosis of the coronary arteries. Mitral and tricuspid regurgitation, $2^{\text {nd }}-3^{\text {rd }}$ degree. Autoimmune hemolytic anaemia, immune thrombocytopenia (Evans' syndrome). Hypertensive heart disease. Heart failure NYHA class III. Artificial pacemaker since 2006, replaced in 2008. Secondary pulmonary hypertension. Cardiac liver fibrosis. Steroid osteoporosis. L3 vertebral compression fracture.

\section{Discussion}

The diagnosis of AHA was based on the following laboratory findings:

- normochromic anaemia (MCH 27 pg);

- moderate reticulocytosis (up to 37\%o);

- presence of erytronormoblasts in the blood (2/100 erythrocytes);

- hyperbilirubinemia (total bilirubin $50 \mu \mathrm{mol} / \mathrm{L}$ );

- high LDH levels (519-1200 IU/L);

- spleen enlargement;

- ruling out other anaemia mechanisms.

The autoimmune HA nature was indicated by the identification of $\operatorname{Ig} G$ autoantibodies on the surface of red blood cells (positive direct Coombs' test dated December 02, 2015).

One of the features is the advanced age of the patient ( 83 years old at the time of diagnosis). The maximum age of the patients followed up by Evans was 78 years, and the average age of 68 patients in another follow-up study was $55 \pm 33$ years ${ }^{6}$. For more than 10 years after detection of cytopenia, the patient did not have signs of disease (lymphoproliferative process, malignant tumor etc), that would have to be ruled out in the presence of autoimmune cytopenias (AHA, ITP). When monitoring the patient, deterioration of the condition (increased weakness, heart failure severity, symptoms of cerebral ischemia), backed up by a decrease in hemoglobin and platelet parameters affecting the quality of life and requiring hospitalization, was noticed. It is shown that the risk of cardiovascular complications in patients with ES is higher than the complications of anaemia or ITP. Among 28 patients $>60$ years old, at the time of AHA diagnosis, cardiovascular complications were observed in 6 patients (21.5\%), including myocardial infarction (1), acute coronary syndrome (4), and stroke (1) ${ }^{6}$.

It is considered that ES develops due to the immune system dysfunction, with appearance of an abnormal clone of immunocompetent cells that produce autoantibodies to their own red blood cells and platelets. In recent years, research on the role of tripeptidyl peptidase deficit, which is revealed in patients with ES, and susceptibility to viral infections have been performed? There is evidence of the immune ITP and AHA development in children after vaccination $^{8}$, suggesting a possible role of immunization in initiating the disease mechanisms in people with genetic predisposition ${ }^{9,10}$.

There is a distinction between idiopathic and symptomatic forms of ES. The latter is seen in 
rheumatoid arthritis, systemic lupus erythematosus, antiphospholipid syndrome, chronic hepatitis, chronic lymphocytic leukemia, malignant lymphomas, and tuberculosis ${ }^{11-13}$. Sometimes, ES is the clinical laboratory onset of the aforementioned diseases. The idiopathic form does not show a clear connection between hemolysis/ ITP and any other pathological process. In one of the largest studies, involving 68 adult patients with ES, idiopathic and symptomatic forms were found with the same frequency ${ }^{6}$.

One of the AHA features in the patient presented is the lack of pronounced reticulocytosis (the maximum values are $37 \%$ ) with a persistent hemolysis, confirmed by a positive Coombs' test. In some cases of chronic immune hemolysis, reticulocytosis may be missing, for various reasons. One reason may be the presence of autoantibodies not only to mature red blood cells, but also to "newborn erythrocytes" (reticulocytes), and even to cells of erythroid bone marrow $^{14}$. Also, the bone marrow hematopoiesis in old and senile age is affected by certain impairments, as illustrated by the so-called myelodysplastic syndrome, with a dysfunction and failure to respond adequately to the reduced number of erythrocytes, in situations such as blood loss or hemolysis. For example, the development of myelodysplastic syndrome occurred in two cases among 68 monitored patients with $\mathrm{ES}^{6}$.

The patient was found to have a monoclonal protein (MP), which is a laboratory marker of the presence of a clone of immunocompetent cells synthesizing MP (heavy or light chains). The latter is detected in lymphoproliferative tumors (multiple myeloma, chronic lymphocytic leukemia), autoimmune diseases (AHA, systemic lupus erythematosus, hepatitis) or found in the absence of the aforementioned diseases, especially in elderly and senile persons. Thus, among out-patients aged 62-95 years, MP was detected in 11 of $111(10 \%)$ at a concentration of 0.2-1.8 g/dL, while in patients aged $<80$ years, MP was detected in $6 \%$ cases, among centenarians ( $>90$ years old) the detection rate reached $14 \%{ }^{15}$. In our patient, the association of MP with the presence of ES reflects immune dysregulation, against which autoimmune cytopenias develop, in particular ES.

The first-line drugs for treating ES are glucocorticoids (GC), and in the absence of their effect, immunoglobulin preparations for intravenous administration. Second-line treatments include immunosuppressants (cyclosporin), monoclonal antibodies (rituximab), splenectomy. The third line includes cytostatics (cyclophosphamide), thrombopoietin receptor agonists (with ITP prevalence), allogenic or autologous stem cell transplantation ${ }^{16,17}$.

In our patient, attention is drawn to the duration of the disease and the fact that for nearly ten years the cytopenia was not treated, and only in 2015 GC were prescribed. ES is characterized by varied progression and unequal treatment response, may occur transiently, chronically, and recurrently. Upon prolonged follow-up (an average of 4.8 years) of patients with ES, it was found that along with a full or partial remission upon treatment termination (32\% of patients) or when treated with GC and (or) immunosuppressants (56\% of patients), 8 patients $(12 \%)$ had the persisting laboratory signs of cytope$n^{2}{ }^{6}$. Despite the ongoing glucocorticoid therapy, our patient had persistent anaemia and ITP with fluctuations in laboratory parameters (Fig. 1) and related clinical manifestations (anaemia, recurrent hemorrhagic syndrome). The special clinical feature of the patient is back pain and reduced mobility due to the lumbar vertebral fracture. This syndrome, disrupting the patient's quality of life, was iatrogenic in nature, because of steroid osteoporosis against the background of prolonged GC administration. Steroid osteoporosis is known as one of the iatrogenic complications of glucocorticoid therapy, developing in $30-50 \%$ of patients receiving $\mathrm{GC}^{18}$

\section{Conclusions}

ES is rare in elderly patients. Thus, the presence of anaemia and ITP in elderly patients require ruling out diseases frequently encountered in elderly patients, such as $B_{12}$ deficiency anaemia, certain forms of hematological malignancies (chronic lymphocytic leukemia with autoimmune cytopenias), myelodysplastic syndrome, iron deficiency anaemia secondary to gastrointestinal tumors, paraneoplastic hematologic syndromes etc. The drug genesis of cytopenia is not ruled out, considering the forced polypragmasy in the elderly patients. Meanwhile, the presence of such a hematological syndrome in these patients not only contributes to the clinical pattern of poly-morbidity (heart failure, encephalopathy etc.), but also complicates the diagnosis search. ES, having immune pathogenesis, requires an active therapy (GC, immunosuppressants, splenectomy), with a high risk of iatrogenic events (infections, steroid ulcers, arterial hypertension, diabetes mellitus etc). Prolonged GC treatment results in situations when a disease for which GC is prescribed fades into the background, and the main manifestations are caused by GC therapy complications. The arising complications impair the quality of life to a much greater extent and become the second (acquired) disease, as was the case in this patient. This should be considered when choosing the appropriate treatment for elderly patients with ES, taking into account the possible benefits, for one part, and the risks of iatrogenic events, for the other . 


\section{Compliance with Ethics Requirements:}

„The authors declare no conflict of interest regarding this article"

„The authors declare that all the procedures and experiments of this study respect the ethical standards in the Helsinki Declaration of 1975, as revised in 2008(5), as well as the national law. Informed consent was obtained from the patient included in the study"

"No funding for this study"

\section{REFERENCES}

1. Evans RS, Takahashi K, Duane RT, Payne R, Lie CK. Primary thrombocytopenic purpura and acquired hemolytic anaemia: evidence for common etiology. Arch Intern Med 1951;87:48-65.

2. Cimá-Castañeda MA, Ayala-López PM, Lara-Palacios MI, Abblitt-Luengas SM, Jiménez-Báez MV. Síndrome de Fisher-Evans o de Evans. Rev Haematol Mex. 2016;17(2):144-149.

3. Silverstein MN, Heck FJ. Acquired haemolytic anaemia and associated thrombocytopenic purpura: with special reference to Evans syndrome. Mayo Clinic Proceedings 1962;37:122-128

4. Pui C, Wilimas J, Wang W. Evans syndrome in childhood. The Journal of Pediatrics 1980; 97: 754-758.

5. Cai JR, Yu QZ, Zhang FQ. Autoimmune haemolytic anaemia: clinical analysis of 100 cases. Zhonghua Nei Ke Za Zhi. 1989;28(11):670-673.

6. Michel M, Chanet V, Dechartres A, et al. The spectrum of Evans syndrome in adults: new insight into the disease based on the analysis of 68 cases. Blood 2009;114:3167-3172.

7. Zhukovskaya EV, Teplykh EV, Basharova EV, Spichak II. Combined immunosuppressive therapy for the Fischer
Evans syndrome in children. Pediatric Gazette of the Southern Urals 2015;1:67-69

8. Norton A, Roberts I. Management of Evans syndrome. British Journal of Haematology 2005; 132: 125-137.

9. Ahmed FE, Albakrah MS. Neonatal familial Evans syndrome associated with joint hypermobility and mitral valve regurgitation in three siblings in a Saudi Arab family. Ann Saudi Med. 2009; 29(3):227-230.

10. McLeod AG, Pai M, Carter RF, Squire J, Barr RD. Familial Evans syndrome: a report of an affected sibship. J Pediatr Haematol Oncol. 1999; 21(3):244-247.

11. Iftimie G, Bratu OG, Socea B, et al. Pulmonary involvement in rheumatoid arthritis - another face of the coin. Arch Balk Med Union 2018;53(1):89-95.

12. Diaconescu D, Pantea Stoian A, Socea L, et al. Hepato-renal syndrome: a review. Arch Balk Med Union 2018;53(2):239-245.

13. Paraschiv B, Dediu G, Iancu A, Bratu O, Diaconu C. Superior vena cava syndrome - review. Arch Balk Med Union 2017;52(1):39-43.

14. Roush GR, Rosenthal NS, Gerson SL, et al. An unusual case of autoimmune haemolytic anaemia with reticulocytopenia, erythroid dysplasia, and an IgG2 autoanti-U. Transfusion 1996; 36: 575.

15. Crawford J, Eye MK, Cohen HJ. Evaluation of monoclonal gammopathies in the "well" elderly. Am J Med. 1987;82(1):39-45.

16. Santiago-Ríos KJ, Reyes O, Cruz A, Rodríguez-Pabón N, Cáceres W. Adult Evans syndrome: complete haematologic recovery with steroids and rituximab: a case report. Bol Asoc Med PR. 2010;102(2):33-6.

17. Jaime-Pérez JC, Elva P, Calderón A, Salazar-Cavazos L, Gómez-Almaguer D. Evans syndrome: clinical perspectives, biological insights and treatment modalities. Journal of Blood Medicine 2018:9 171-184.

18. Briot K, Roux C. Drug-induced osteoporosis: beyond glucocorticoids. Curr Rheumatol Rep. 2008;10(2):102-109. 